\title{
Non destructive evaluation of absorbing materials using microwave stimulated infrared thermography
}

\author{
by P.LEVESQUE, A.DEOM AND D.BALAGEAS
}

$(*)$

(*)ONERA, Thermophysics Division, BP 72 , F-92322 CHATILLON-Cedex, France.

\begin{abstract}
The electromagnetic wave non destructive evaluation methods that appeared some years ago are attractive because many materials can absorb hyperfrequency energy. A new method was developed which associates a microwave stimulation and an infrared detection by IR thermography. An experimental $10 \mathrm{GHz}$ setup was built to verify the feasability of the method. Results are shown of the use of this method for non destructive evaluation of absorbing materials containing artificial defects.
\end{abstract}

\section{Introduction}

The electromagnetic wave nondestructive evaluation methods that appeared some years ago are attractive because many materials can absorb hyperfrequency energy. Nevertheless, generally detections are achieved point by point which is highly time consuming for non destructive evaluation of extended structures. We developed a global method which associates an ElectroMagnetic stimulation and a detection by an IR camera (EMIR method). For others applications, this method has been used over the past several years [1,2]. The aim of this paper is to give the first results about the possibilities of this new method for NDE.

\section{Experimental set-up}

For experiments the entire set-up is placed in an anechoic chamber, $7 \mathrm{~m}$ long and $4 \mathrm{~m}$ large in order to minimize the reflections of the electromagnetic waves. The excitation of the samples is produced by horns or parabolic antennas in the $8-18 \mathrm{GHz}$ range ( $\mathrm{X}$ and $\mathrm{KU}$ bands). An amplifier with power up to $100 \mathrm{~W}$ is used to excite the antennas. The front face temperature of the samples is monitored by an infrared camera in the 8-12 $\mu \mathrm{m}$ range (AGEMA, model 782 LWB).

The excited area is about $30 \times 30 \mathrm{~cm}$. It is very easy to change the dimensions of the inspected area only by changing the objectives of the IR camera. Typically in the present application this area ranges from $10 \times 10$ to $30 \times 30 \mathrm{~cm}$. Depending on the samples and the applications, the incident power density on the samples varies from some 10 to $100 \mathrm{~mW} / \mathrm{cm}^{2}$.

The signals delivered by the camera are digitized and a real time storage is achieved by a CEDIP PTR-9010 system hosted by a microcomputer Compaq 386 . It is then possible to record all the frames delivered by the camera ( 25 frames/s) with 12 bit resolution and a definition of the frames of 64 lignes of 128 points directly on the hard-disk of the computer for time durations up to $20 \mathrm{~min}$. The distance between the antennas and the samples is a few meters.

\section{Specimens}

Specific samples were built to test the method. They consist of absorbing materials containing inserts of different electrical conductivity, with different sizes and at different depth location.

The samples are glass-epoxy composites loaded with carbon particles. The resulting electrical conductivity of the bulk material is $\sigma=5 \Omega^{-1} \cdot \mathrm{m}^{-1}$. Two types of inserts are used : highly conductive materials consisting of stainless sheets $\left(\sigma=1.410^{10} \Omega^{-1} \cdot \mathrm{m}^{-1}\right)$ and lower conductive materials made of the same material as the bulk but with a higher load in carbon particles 
$\left(\sigma=25 \Omega^{-1} \cdot \mathrm{m}^{-1}\right.$.). Figure 1 shows inserts distribution in a test sample. For this material, the penetration depth, at $10 \mathrm{GHz}$, is $4.3 \mathrm{~mm}$ so the insert at $7.68 \mathrm{~mm}$ must be difficult to detect.

These samples, which are $10 \mathrm{~mm}$ thick, are optimised in order to be used with a metallic sample behind their rear face so that they cannot be evaluated using a transmission method.

\section{Experimental results}

\subsection{Normalisation of the infrared Images}

Figure 2 shows the infrared image of a reference sample (glass epoxy plate without defect) with a steady-state excitation. This image is recorded $20 \mathrm{~s}$ after the beginning of the excitation. The incident power is about $100 \mathrm{~mW} / \mathrm{cm}^{2}$. The sample heating is proved to be strongly non uniform and this is due to the use of a horn and a short distance between the antenna and the sample (near-field of the horn). The temperature increase at the center of the sample is about $1.5^{\circ} \mathrm{C}$ with respect to the temperature of the cold areas.

An image recorded in the same conditions on a sample with inserts (figure 3) shows roughly the same thermal map with little disturbances at the location of three of the defects. An image processing is obviously necessary to remove the non-uniformity of the excitation.

Figure 4 and figure $A^{*}$ show the result of a simple subtraction between the two previous images (absolute contrast image). The defects are more visible and they can be seen up to $5.76 \mathrm{~mm}$ in depth. In order to be independent of the amplitude of the source, it is better to use the relative contrast :

$$
\Delta \theta=\left(\Delta \theta_{a d}-\Delta \theta_{s d}\right) / \Delta \theta_{s d}
$$

with $\Delta \theta_{a d}$ the plate temperature with defects, and $\Delta \theta_{s d}$ the reference plate temperature. This normalisation is made pixel by pixel.

The efficiency of the normalisation is validated by comparing several tests made with different excitations. Table 1 shows the relative contrast at the center of defects $n^{\circ} 1,2$ and $3 . P$ is the outpout power of the amplifier, $d$ the distance between the antenna (horn or parabolic antenna) and the sample.

The small discrepancy observed in the results is not important considering the large change in the experimental conditions.

\subsection{Sensitivity of the method to inserts characteristics}

A measurement with a stainless sheet insert, $10 \times 10 \mathrm{~mm}$ size, shows no decrease of the relative temperature contrast in spite of the decrease of the insert size (previous results with $20 \times 20 \mathrm{~mm}$ ).

Figure 5 shows also no obvious variation of the relative temperature increase with the thickness of the stainless sheet in the range 25 to $100 \mu \mathrm{m}$. The influence of the thickness becomes visible when it becomes of the order of few micrometers.

A change in the electrical conductivity of the insert leads to a change in the relative temperature contrast at the insert location (figure 6). In this figure one can remark also the shape of the insert at the lower depth certainly due to a slip of the carbon powder during the processing of the plate.

An experiment with an insert with $\sigma=25 \Omega^{-1} \cdot \mathrm{m}^{-1}$ allows to verify that the lower the difference in the electrical conductivity between the insert and the bulk material, the lower the thermal contrast. Table 2 shows the measured variation of the maximum detectable depth with the characteristics of the inserts.

\subsection{Relative contrast of the defects}

It is possible to calculate the volumic power absorption and then the temperature increase at every point inside the reference plate, assuming that its thickness is much larger than the penetration depth (figure 7). Such a calculation is also possible for a plate containing large inserts assuming that the defect has an infinite lateral extension. Since experiments are performed in 
steady-state regime there is a balance between the absorbed power and heat losses (heat tranfert coefficient $h$ ) which gives the temperature increase $\Delta T$. It is then possible to compare the experimental and calculated relative temperature contrasts ratio between two defects. Table 3 shows such a comparison for the plate with $20 \times 20 \mathrm{~mm}$ stainless sheets inserts, $50 \mu \mathrm{m}$ thick. $\Delta T_{1}$, $\Delta T_{2}$ and $\Delta T_{3}$ are respectively the relative contrast in temperature at the location of inserts 1,2 and 3 at respective depths $1.92,3.84$ and $5.76 \mathrm{~mm}$. The frequency used for the measurements is $10 \mathrm{GHz}$. The quite good agreement between theory and experiments proves that the normalisation is correct.

\section{Improvement of the method by the use of a film detector}

The previous method, associating microwave excitation of a sample and a monitoring of the front face heating allows to detect with some quantification local electrical conductivity non uniformities of the sample. But it needs a reference sample and a high power microwave source.

An other method in progress in the laboratory consists to use a microwave thermal detector film which is placed at a given and small distance of the front surface of the inspected sample. The film allows to observe the pertubation of the incident electromagnetic field due to the presence of a non uniformity in the electrical conductivity of the structure. This method requires a lower incident power density than the previous method and gives a higher signal to noise ratio in defect detection. The principle of the two EMIR methods is given in figure 8 .

Figure $B$ shows an infrared image obtained with the film detector. The sample is the same as the one previously used (figures 1 to 4). The presence of the inserts can be detected without any reference plate or normalisation, and with a reduced power : $17 \mathrm{~mW} / \mathrm{cm}^{2}$. The power density reduction is of a factor of 5 .

\section{Conclusion}

A NDE method mixing microwave excitation ( $X$ and $K U$ bands) and infrared thermographic detection (EMIR method) on the front face of the structure to inspect was investigated.

The method applies to dielectric or conductive materials with conductive defects.

The capabilities of the method were illustrated by characterizing artificial defects consisting in small inserts of various electrical conductivities and different sizes, located at different depths in composite materials samples. It was possible to detect electrical conductivity differences between the inserts and the bulk material ranging from a few $\Omega^{-1} \cdot \mathrm{m}^{-1}$ to $10^{6} \Omega^{-1} \cdot \mathrm{m}^{-1}$, at depths of a few millimeters, depending on the ratio between the two conductivities.

A quantitative validation of the method was done by comparing experimental temperature images obtained by the infrared camera and theory. A quite good agreement was obtained, in particular with highly conductive defects.

The weakness of the method is the necessity to use relatively large electromagnetic power density $\left(100 \mathrm{~mW} / \mathrm{cm}^{2}\right)$.

A new method is in progress in our laboratory using a microwave thermal detector film, which allows to reduce by a factor of five the power density. Enhancement of the sensitivity and elimination of free convection perturbations could be possible by using both an amplitude modulated source and a lock-in infrared thermography. First results recently obtained seem to be very promising.

\section{Acknowledgements} (STPA/MA). 


\section{REFERENCES}

[1] SEGA (R.M.) and NORGARD (J.D.). - Infrared measurements of scattering and electromagnetic penetration through apertures. EEE Transactions on Nuclear Sc., Vol.33, $\mathrm{n}^{\circ} 6$, 1986.

[2] NORGARD (J.D.) and SEGA (R.M.). - Microwave fields determined from thermal patterns. SPIE, Vol. 780, Thermosense IX 1987.

\begin{tabular}{|c|c|c|c|}
\hline $\begin{array}{c}\text { Experimental } \\
\text { set-up }\end{array}$ & Defect $n^{\circ}$ & Defect $\mathrm{n}^{\circ} 2$ & DSefect $\mathrm{n}^{\circ} 3$ \\
\hline $\begin{array}{c}\text { horn } \\
d=40 \mathrm{~cm}\end{array}$ & 0.271 & 0.098 & 0.240 \\
\hline $\begin{array}{c}\text { parabola } \\
d=2.2 \mathrm{~m}\end{array}$ & 0.235 & 0.094 & 0.184 \\
\hline $\begin{array}{l}P=63 \mathrm{~W} \\
\text { parabola } \\
d=2.2 \mathrm{~m} \\
P=83 \mathrm{~W}\end{array}$ & 0.360 & 0.076 & 0.207 \\
\hline
\end{tabular}

Table 1 - Comparison between the relative temperature contrasts.

\begin{tabular}{|c|c|c|c|c|c|c|}
\hline $\begin{array}{c}\text { Ratio of the electrica conductivities } \\
\text { of the defect and the bulk material }\end{array}$ & \multicolumn{3}{|c|}{$10^{6}$} & 20 & 5 \\
\hline Square defect dimension $(\mathrm{mm})$ & 5 & \multicolumn{2}{|c|}{10} & 20 & 10 & 10 \\
\hline Defect thickness $(\mu \mathrm{m})$ & 50 & 50 & $\begin{array}{r}50 \\
25\end{array}$ & 50 & 500 & 500 \\
\hline Maximum detectable depth $(\mathrm{mm})$ & 5.76 & 5.76 & 5.76 & 5.76 & 3.75 & 1.75 \\
\hline
\end{tabular}

Table 2 - Variation of the maximum detectable depth with the characteristics of the inserts for a power density roughly equal to $100 \mathrm{~mW} / \mathrm{cm}^{2}$ at $10 \mathrm{GHz}$.

\begin{tabular}{|c|c|c|}
\hline $\begin{array}{c}\text { Relative temperature } \\
\text { contrast values }\end{array}$ & $\begin{array}{c}\text { Experience } \\
\text { (steady-state) }\end{array}$ & $\begin{array}{c}\text { Theory } \\
\left(\int P d x / h\right)_{1} /(f P d x / h)_{2}\end{array}$ \\
\hline$\Delta \mathrm{T}_{1} / \Delta \mathrm{T}_{2}$ & 1.66 & 1.62 \\
\hline$\Delta \mathrm{T}_{1} / \Delta \mathrm{T}_{3}$ & 1.22 & 1.30 \\
\hline$\Delta \mathrm{T}_{3} / \Delta \mathrm{T}_{2}$ & 1.36 & 1.24 \\
\hline
\end{tabular}

Table 3 : Comparison of experimental and theoretical values of the relative thermal contrast at location of inserts. 
http://dx.doi.org/10.21611/qirt.1992.047

Steady-state regime heating $\left.\quad \mathrm{T}^{\circ} \mathrm{C}\right)$

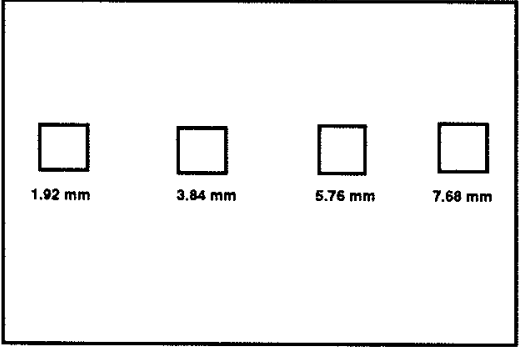

Fig.1. - Inserts distibution in test sample.
Steady-state regime heating

$\mathrm{T}\left({ }^{\circ} \mathrm{C}\right)$

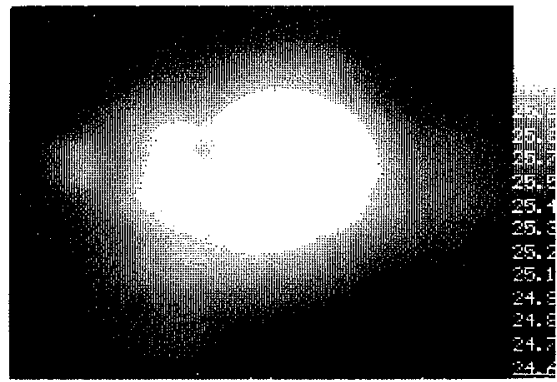

Fig. 3. - Infrared image of a sample with defects.

Relative temperature contrast in steady-state regime

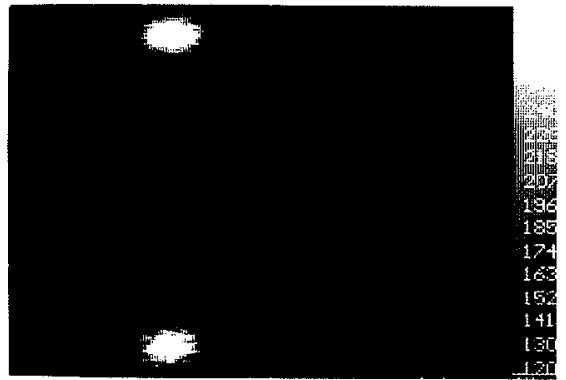

Fig. 5. - Sample with two defects of different thickness at same depth.: $A=25 \mu \mathrm{m}$ and $B=100 \mu \mathrm{m}$.

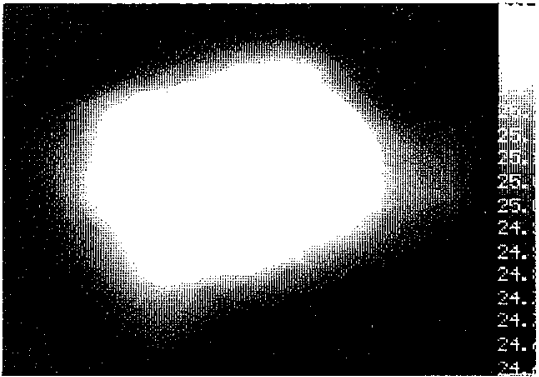

Fig. 2. - Infrared image of the reference sample.

\section{Absolute temperature contrast in $\quad \Delta T\left({ }^{\circ} \mathrm{C} \times 1000\right)$ steady-state regime}

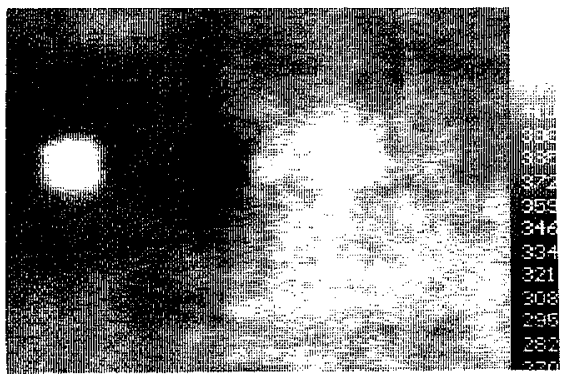

Fig. 4. - Result of a simple subtraction between the two images of fig. 2 and 3

\section{Relative temperature contrast in} steady-state regime

(\%)

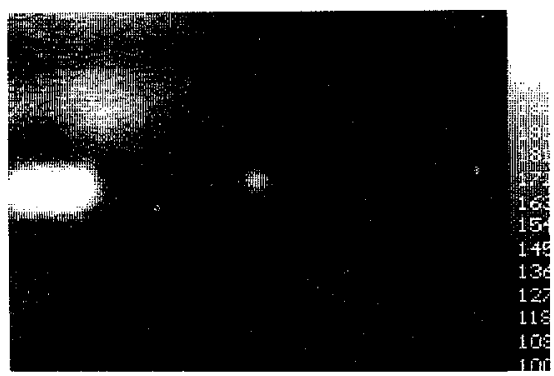

Fig. 6. - Defects with electrical conductivity of $100 \Omega^{-1} \cdot \mathrm{m}^{-1}$. The large defect is located at $1.92 \mathrm{~mm}$. 


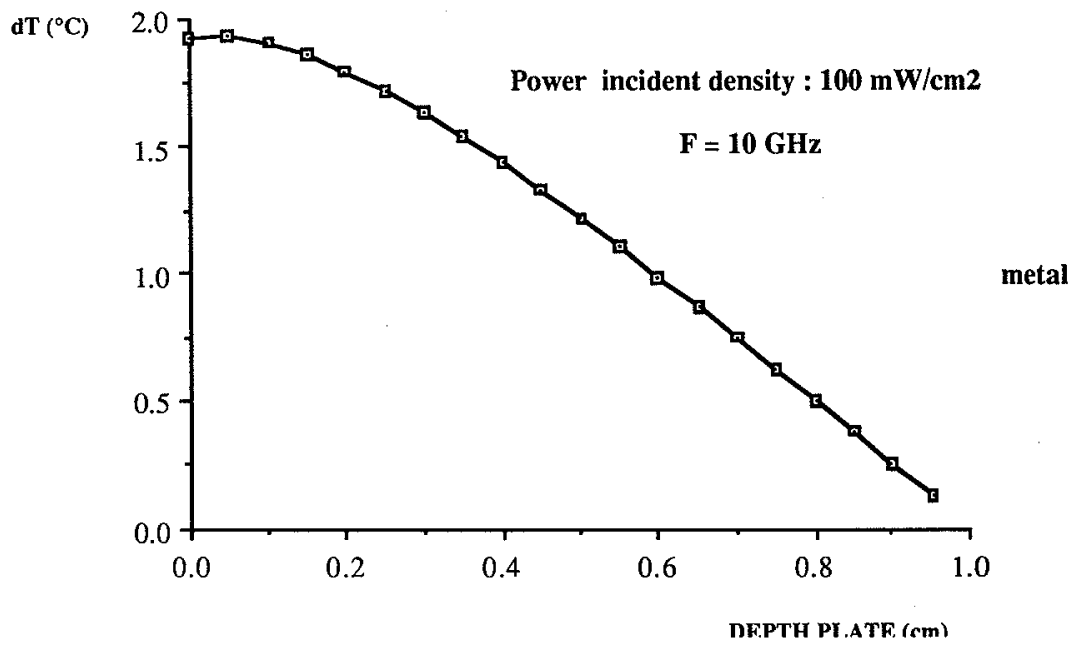

Fig. 7. - Theoretical temperature profile in reference plate $(10 \mathrm{~mm}$ thick).

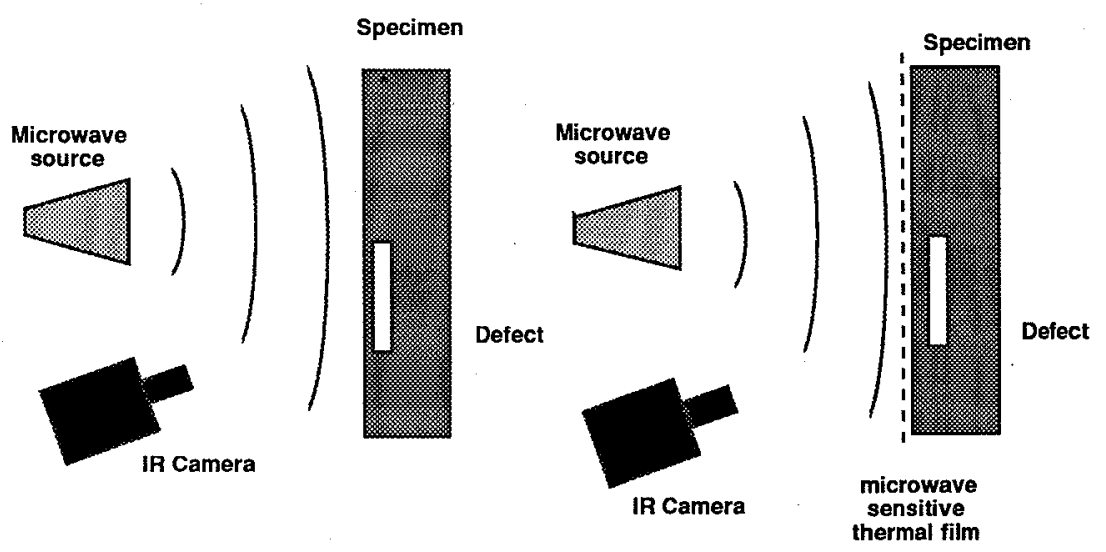

Fig. 8. - Principle of EMIR NDE methods. Left : direct method; Right : film method. 